THE BOLLER REVIEW

\title{
A PHENOMENOLOGICAL ANALYSIS OF DISABILITY AND EMPOWERMENT
}

\author{
MARTIN PTAK
}


A central theme when analyzing quality of life issues is empowerment and how it can ultimately serve as a driving force toward success and fulfillment. In spring 2018, I embarked on a journey into understanding this phenomenon through a phenomenological lens. As an undergraduate, pre-med, biology student, I enrolled in an honors research course led by Dr. Nina Martin from the TCU School of Classical \& Contemporary Dance and Dr. Eric Simanek from the TCU College of Science and Engineering that focused on exploring the effects of an improvisational dance method termed the "Martin ReWire Movement Method" (MRMM) within a group of dancers. The goal of this study was to observe and understand the effects of MRMM within this group of people and its impact on their quality of life. Participants were three dance artists living with cerebral palsy who dance with the Body Shift dance company in Austin, Texas. In this time, I also applied for a research project within this course that would qualify as a colloquium for my honors curriculum (with granted permission from the John V. Roach Honors College). My individual research project focused in understanding how the notion of empowerment functions for individuals living with cerebral palsy.

Cerebral palsy is a non-progressive, neurological disorder which develops shortly before or during birth. While the cause for the disorder is not completely understood, some research has found a link with injury to the cerebral cortex of the brain, which is responsible for muscle coordination and movement (Colver). As researcher Andrew Koman explains, "the characteristic signs [of cerebral palsy] are spasticity, movement disorders, muscle weakness, ataxia, and rigidity. Cerebral palsy is the most common cause of severe physical disability in childhood" (1240). Within the past few years, Dr. Nina Martin has been developing a therapeutic dance practice which appears to have positive effects on participants with cerebral palsy. Primary reports indicated improvements in the participants' spasticity, flexibility, and range of motion after performing the therapeutic dance, later named "Martin ReWire Movement Method (MRMM)." Research into the benefits of dance for adults living with cerebral palsy is an under researched area, which makes resource information and background knowledge limited. To begin studying the effects of MRMM on people with cerebral palsy, the researchers' collected data that allows for scientific exploration and analysis, though my research focuses on the qualitative aspect of this data.

MRMM is an improvisational dance technique centered around spontaneous, non-repetitive and repetitive movements while in a prone or supine position on a floor-mat. The quick, monotonous movements discourage any preplanned movements by having participants execute short, unplanned movements for a set period of time. Furthermore, this supports the participant in not consciously attending to the movement or engaging in predictive behavior. Dr. Martin describes this process as "kill the choreographer" (or the premeditating planner) and instead allow movement to emerge from the body in evolving sequences (each lasting approximately half a second). With this emphasis placed on the disconnect of the conscious mind attending to the body's movements, MRMM allows for the participants to be receptive to their preconscious movement impulses and allow the movements to emerge in an unplanned manner.

At the end of a MRMM practice session, the participants reported experiencing a temporary cessation of spasms and increased range of motion. The dance practice appears to have an effect on the nervous system in the area where people with cerebral palsy suffer the most: motor control. To study this effect, Dr. Martin and Dr. Simanek's class used a two-fold approach: quantitative and qualitative. Quantitatively, the physical effects of the movement method were recorded as measurements in spasticity, flexibility, and range of motion and further quantified using motion tracking software called "Kinovea." In addition to the physical effects of MRMM, a qualitative approach was also required, as participants present certain aspects of emotionality and impressionability as evidenced by some participants who became emotional and began crying or felt ecstatic either during or after the dance session. This paper focuses on the 
value of a phenomenological approach in examining a sampling of the qualitative data supported by literature from the fields of ontology and philosophy.

Empowerment became an apparent theme emerging from the data analysis within this group of individuals after witnessing changes in self-esteem and morale following MRMM. For this research I use "empowerment" as a term that embodies aspects of identity and personal understandings, and is an antithesis of discouragement. A remarkable characteristic of empowerment is its adaptability and capacity to manifest itself in every aspect of one's life. In an English translation of sociologist Anne Calvès' article titled "Empowerment: The History of a Key Concept in Contemporary Development Discourse" she writes, "From popular psychology to self-help, the infatuation with empowerment in the English-speaking world appears boundless: in 1997 there was even a book published in the United States on 'self-empowerment' for dogs" (Calvès 735). The versatility of the term makes it a useful tool for observing several characteristics and support in living a fulfilled life. Furthermore, empowerment can be used as a means of establishing empathetic connection, gaining wisdom within a worldly population, and achieving a fulfilling and satisfied life. However, in order to fully understand the changes and their significance, a deeper qualitative examination into the participants' perceptions of their experiences, character, and perception of the movement method is necessary.

One way to investigate these areas is to use phenomenological research methods. According to the Stanford Encyclopedia of Philosophy, phenomenological research focuses on heuristics, which is described as an area of study with a primary focus on an individual's identity and experiences, as well as philosophy of mind. Phenomenology has been practiced through particular viewpoints for centuries, but officially became recognized as an independent, philosophical method in the early 20th century as articulated by the works of Husserl, Heidegger, Sartre, and others. In Clark Moustakas' book explaining phenomenological research, he explains, "Heuristics is a way of engaging in scientific research through methods and processes aimed at discovery; a way of self-inquiry and dialogue with others aimed at finding the underlying meanings of important human experiences" (18). My own self-reflection and dialogue are research methods that were utilized throughout this study. During the first data gathering trip, Quality of Life (QOL) surveys were filled out by the participants which gaged their personas in categories of well-being, depression, anxiety, and fatigue. However, not all of the questions were applicable or answered within the context intended by the survey for the participant.

As a result, Dr. Martin and I returned to Austin, Texas (supported by a research grant from the John V. Roach Honors College) to meet with the MRMM participants to gather additional data based on dialogue and interaction in open-ended interviews. Open-ended interviews are an important data-gathering technique because they allow for participants to share in the directionality of the conversation based on interests and passion. The importance of dialogue within phenomenological research is well explained in Moustakas' book. He writes, "Phenomenology, stepby-step, attempts to eliminate everything that represents a prejudgment, setting aside presuppositions, and reaching a transcendental state of freshness and openness..." (41). Having a conversation with someone allows a new perspective to be assessed. When questions can only be answered using provided options such as "yes," "no," or "sometimes," a void of explanation occurs which potentially holds fundamental value in empowerment and identity research. Thus, group-style discussion has become an evident tactic in deriving valuable data sets. For this study, I devised a list of several open-ended interview questions (see Appendix 1) to guide a conversation with each participant. Open-ended questions allow for deeper understanding in areas of mental states, temperament, and perception. Moustakas explains that heuristic research proceeds through several phases which comprise the basic research design. The beginning phases include initial engagement, immersion into the topic and question, incubation, illumination, and explication (18). When giving participants some freedom to guide the conversation, one can assess the perceived importance of 
the discussed topic based on how long or short the exchange is. Initial engagement is accomplished by delivering the interview question followed by other questions for clarification and explanation. This also shows the participant's engagement level and genuine interest on behalf of the interviewer in what they have to say; this leads to more accurate analysis of the data in preparation for the steps of illumination and explication.

Establishing a comfortable baseline proves critical when starting conversations that can often lead to deep and thought-provoking discussion. Another important aspect to consider for these interviews was question complexity. Without a gradual increase in question complexity throughout the interview, the participants may become intimidated or struggle to immediately formulate an answer that correctly conveys their ideas on the subject of a deep question. This is why the first few questions about the participants' upbringings were important in not only understanding what kind of experiences have influenced the person they have become, but also in requiring simple recall to answer the questions. "In phenomenological research, the question grows out of an intense interest in a particular problem or topic...Personal history brings the core of the problem into focus" (Moustakas 105). Working from childhood experiences to present day experiences offers a conceptual timeline to see how the participants have evolved as individuals, often times exposing aspects of their growth in mentality and sense of reality. Becoming an empowered individual is often influenced by one's past experiences, while also being cultivated by an individual's community or support system.

There are several important discoveries I made from the interviews with the participants living with cerebral palsy. Most of these discoveries center around the participants' strong sense of purpose and self-discovery. During the interviews, each participant explained that the early years of their life were filled with uncertainty and fear. Such feelings stem from not knowing the full extent of their disability at a young age, given that cerebral palsy can occur in varying degrees of severity and may be diagnosed only when certain developmental benchmarks are not met. This early time seemed to be vital in establishing a strong support system within their surrounding group of family and friends. Strong support systems initiate an empowered mindset of knowing that these individuals are not alone during a difficult time in their lives, and also finding support during their ongoing rehabilitative and therapeutic practices.

Dr. Li Li of New York University in her article for The Journal of Social Psychology explains that, "Acceptance of disability is directly associated with the success of rehabilitation. That improved self-esteem, reliable emotional support from family and friends, and reduction of the stigmatizing effects of disability could positively impact the processes of adjustment and rehabilitation" (23). In this way, I noticed the acceptance of disability by the participant became another important aspect of self-empowerment. Following this idea, each participant also reported that the supportive environments during childhood consisted of making sure that the participants were taught how to adapt to their environment given their disability. This was a key aspect in understanding why every participant, during their interview, expressed one of their hallmark traits as being a "good problem-solver." Elena Morozova suggests people with high acceptance of their disabilities become adaptive problem-solvers capable of "self-actualization" in her article Acceptance of Disability: Determinants of Overcoming Social Frustration (321). Being adaptive and accepting of their disabilities enables the participants to approach problems with a new perspective, and "outside-the-box" thinking can be seen as a fundamental means for becoming an empowered individual.

Morozova's research leads to another important aspect of empowerment which is a sense of reality. I have termed this sense of reality as "self-reality" in order to emphasize the participants' awareness of their personal circumstances and conditions. This aspect of self-awareness within these participants with cerebral palsy was evident from the interviews conducted. Each participant described that they had established a strong sense of self-reality at a young 
age (pre-pubescent). Their initial growth of self-reality started when the participants' parents would integrate them into school systems with non-disabled children. The reality of their conditions was something that their parents did not want to hide from them by schooling them with other disabled students. Self-reality seems to play a role within the developing state of empowerment that builds a strong foundation of identity, setting personal aspirations, and developing a sense of ultimate purpose further along in life. Li suggests in her 1998 article titled: "Acceptance of Disability and its Correlates" that "traditionally, people with disabilities, like other minority groups, are isolated and segregated from society. Societal integration for people with disabilities requires self-empowerment in addition to mastery of the problems posed by external discrimination" (21). Social integration also helps individuals with disabilities find their own niche within society.

Participants expressed their strong sense of belonging within a disability culture which holds its own set of principles and values aside from broader, societal culture. An accurate sense of self-reality can also prevent an individual's development of unrealistic expectations and hopes. Setting goals that are attainable allows for growth as a person and motivates empowerment. However, in order to determine what is and is not attainable requires understanding of one's reality. Morozova concludes her research of disability acceptance by writing, "investigation of the internal picture of disability of persons with different levels of social frustration has revealed significant personal phenomena that allow one to establish the fact of personal transformation on the basic parameters of selfawareness..." (322). Self-awareness, for this research, occurs in contexts of reality, but also in a physical context with relation to the body.

Empowerment seems to arise from a holistic viewpoint of the participant at the end of MRMM as they can better perform physical actions and have a general sense of well-being. Thomas Hanna, author of The Body of Life, describes this holistic viewpoint as a person's "soma." He writes, "In Greek, the word soma evolved to mean 'the living body in its wholeness'... The soma arose as a system that always strives to achieve stability and balance - a task that takes place in time and is never complete" (5). Each participant with cerebral palsy expressed in their interviews how their bodies were ever changing. While cerebral palsy is a non-progressive disorder, each day their bodies react differently based on environmental factors and secondary complications from such factors as weather, energy levels, overall health, and exercise. Therefore, stability and balance are ever-evolving characteristics of their bodies with which they have to become reacquainted with daily. One participant expressed how MRMM allowed her to do this and become "familiar with [her] body."

The benefits of MRMM accrue due to a seemingly necessary disconnect between the conscious mind and the body's movements by avoiding any kind of premeditation or movement planning during MRMM that occurs in different phases with relation to time. At different phases during the movement method, certain movements are random and others are repetitive, all whilst allowing the body to carry out apparent, natural motions. Hanna conveys a similar concept and review of the relationship between mind, body, and time. He writes:

The marvelous feature of timing is that it embraces the whole of the available soma. Our own consciousness is not so watchful; it [is] always switching about, focusing on this thing and that, never able to take in the wholeness and the spherical completeness of our living body. If the operations of our own organic being were solely dependent upon our consciousness, we would not last a day... (Hanna 120).

Most individuals would think that our consciousness is solely reliable for our survival, and to a certain extent it is. For example, one can determine when to eat based on the known feeling of hunger. However, there are just as many instances when one does not need to think in order to survive. An example is when one takes his/her first breath as a 
newborn. From that moment on, the autonomic nervous system becomes responsible for preserving a person's life and the act of breathing does not need to be consciously activated or controlled; it just happens.

Similarly, MRMM allows for the participant to be receptive to pre-conscious movement impulses and allow them to emerge much like turning over in your sleep. The random movements practiced in MRMM seem to relieve stress or the constant flexion of certain muscles that is an attribute of cerebral palsy. In this way, further research is required into MRMM being offered as a less-invasive therapeutic intervention compared to other techniques such as elective surgeries where procedures cut chronically flexed muscles to prevent skeletal deformation and provide mobility and some comfort. Future research is needed to define a relationship between the duration of the movement intervention and the magnitude of its effects.

With a disability such as cerebral palsy, it often times becomes difficult to feel in control of one's body. Loss of control of motor function can be detrimental to one's feelings of self-awareness or even "wholeness." Thomas Hanna explains, "A somatic distortion is not simply a distortion of the body's structure; it is a distortion of our awareness of ourselves in the physical world. In a certain sense, it is a distortion of our body image" (80). Having poor body image can lead to a decrease in self-worth or pride which impedes development of an empowered personality. Being able to become reoriented with one's body after MRMM can improve perceptions of self-control and, in-turn, body image. Even the smallest improvements in control after MRMM can have a large impact in body wholeness and mentality as conveyed by the participants.

Another important aspect of self-awareness leading to perceptions of empowerment within these participants with cerebral palsy is the idea of MRMM (in itself) as a dance form. Including people living with disabilities as valued artists, as in the Body Shift dance company, has become an increasingly popular concept over the past few years. At first, a disabled performer carried a negative stigma based on their limitations and focusing on what they cannot do shadowed by the idea of how they deviate from a "perfect" body. Petra Kuppers has been studying performers with disabilities for years and published her findings in a book, titled Studying Disability Arts and Culture, designed to help people develop an appreciation for disability arts. She writes:

"Dance communicates the inherent sharedness of movement because it can directly focus on forms of embodiment that are stigmatized and equated with inability. To dance is to live and to signal one's self as alive, whether one speaks or not, whether one externalizes expression or not" (Kuppers 114).

When anyone dances, regardless of whether s/he is disabled, they are expressing themselves in an emotional and physical way. The whole-body expression innate to dance is what makes it personable and central to the performing arts.

According to Kuppers, another form of empowerment in the performing arts is the release of ego and an embodied self-confidence coming forward, complemented with certain vulnerability. This vulnerability occurs when a performer shares their emotionality with an audience or when a performer has a moment of newfound self-realization. Kuppers further explains, "Disability dance does make a difference, opens up the repertoire of dreams, allows everybody to feel the aliveness and embodied skill of disabled dance artists" (118). During vulnerable moments, people's strengths and weaknesses are highlighted, which is important in cultivating a personal identity and character. Through the arts, personal identity and character are developed and empower individuals by transcending tendencies for self-doubt or insecurity. Even in adaptations of traditional dances, such as ballet or the Waltz, there is a fresh deliverance of the form with respect to how performers and audiences experience anew traditional dance forms. "Every time a disabled dance artist dances ballet on a stage, the meanings of ballet shift, and the scope of bodies opens 
up...pushing against normativity on many fronts" (Kuppers 119). The push against normativity does not come without counter actions or criticism from certain individuals. However, for performers with cerebral palsy, their empowered view of themselves may embrace their crutches or wheelchair as extensions of their true bodies. Coming to terms with their reality within dance and public performance gives rise to empowerment through their own personal truths and acceptance of self.

In recent years, the disabled population has become a force to be reckoned with as it embraces a shared empowerment that transcends stigma and discrimination, which can serve as an inspiration to many. The dance world is only one of several different areas in which change is taking place for people with disabilities. The participants with cerebral palsy in this study are all disability activists working to raise awareness for people with all kinds of disabilities. One participant described how she is more interested in bettering the quality of life for all people, rather than just a select few.

James Charlton, in his 1998 book titled Nothing about Us without Us: Disability Oppression and Empowerment, wrote about the psychology and understanding of disability culture and activism. He explains part of this phenomenon when he writes, "Out of similar and divergent experiences, people with disabilities have acquired a consciousness of themselves and the world around them. This new understanding has affected their aspirations and responsibilities... they have become empowered. They no longer think of disability as a medical condition but as a human condition" (115). This newfound consciousness results in part from a strong sense of self-empowerment that propels the disabled into a rightful inclusion in public life. Charlton continues, "persons with empowered consciousness may still see only part of the larger world but understand they can and should influence it. This does not mean that they want to be leaders. It does mean they want to empower others" (119).

One of the difficult features of phenomenological research when exploring empowerment is being able to take the data gathered and, through data analysis, create a cohesive and relevant model that is well-supported. Choosing which discoveries (emerging from the data) are most pertinent takes time and patience. Moustakas describes this final step in most phenomenological research when he writes, "The final challenge of phenomenological reduction is the construction of a complete textural description of the experience...In the process of explicating the phenomenon, qualities are recognized and described; every perception is granted equal value..." (Moustakas 96). Interpreting this kind of data involves case-by-case understanding that not everyone's experiences are the same - including the researcher's. However, drawing connections and finding similarities, while also acknowledging differences, is significant and meaningful.

Through this phenomenological research project my understanding of the relationship between mind, body, and cognition grew, and the potential lifelong benefits of Dr. Martin's ReWire Movement Method within a select population of individuals becomes evident and essential. During this research process, I found myself being inspired by the participants living with cerebral palsy. Their resolve and determination to live a full and meaningful life can serve as a lesson for all. With the help of Dr. Nina Martin, Dr. Eric Simanek, and the support of the TCU John V. Roach Honors College, I was able to discover and better understand the forces of empowerment within a population that changed the way I see my life and the world. 


\section{Works Cited}

Calvès, Anne-Emmanuèle. "Empowerment: Généalogie d'un Concept Clé du Discours Contemporain Sur le Développement." Revue Tiers Monde 4 (2009): 735-749.

Colver, Allan, Charles Fairhurst, and Peter Pharoah. "Cerebral Palsy." The Lancet, vol. 383, no. 9924, 2014, pp. 1240-1249.

Charlton, James I. Nothing About Us Without Us: Disability Oppression and Empowerment. U of California P, 1998.

Hanna, Thomas. The Body of Life: Creating New Pathways for Sensory Awareness and Fluid Movement. Healing Arts Press, 1993.

Koman, L. A., Beth Paterson Smith, and Jeffrey S. Shilt. "Cerebral Palsy." The Lancet, vol. 363, no. 9421, 2004, pp. 1619-31. doi:10.1016/S0140-6736(04)16207-7

Kuppers, Petra. Studying Disability Arts and Culture: An Introduction. Palgrave Macmillan, 2014.

Li, Li, and Dennis Moore. "Acceptance of Disability and its Correlates." The Journal of Social Psychology, vol. 138, no. 1, 1998, pp. 13-25 doi: 10.1080/00224549809600349

Morozova, Elena Valeryevna., et al. "Acceptance of Disability: Determinants of Overcoming Social Frustration." Global Journal of Health Science, vol. 7, no. 3, 2015, pp. 317. doi: 10.5539/gjhs.v7n3p317

Moustakas, Clark. Phenomenological Research Methods. Sage Publications, 2010. 


\section{APPENDIX 1}

Interview Questions for Participants with researcher Martin Ptak

\section{Upbringing:}

What was your childhood like?

When did you start practicing independence? Autonomy?

When did you start to notice you were different?

Can you describe or share an experience?

\section{Empowerment:}

When do you feel the most empowered?

What do you hope to achieve through your activism? OR

Do you see an end goal for advocacy?

Do you see yourself as being proactive or reactive?

\section{Connection:}

Who do you feel the most connected to in your life? Why?

How would you define your relationship with able bodied people vs those with CP?

\section{Triumphs:}

What are some of your biggest triumphs in your life? OR

What are you most proud of that you've achieved in life?

What do you hope to accomplish in the next years? OR few years?

What are your current goals in life?

\section{Challenges:}

What was your biggest challenge this week?

What has been your biggest challenge in life?

\section{Sense of belonging:}

When do you feel valued? When (or in what circumstances) do you feel the most comfortable to be your true self?

Personal traits and interests:

What are your strengths?

What would your friends and family say your best traits are? OR

If we were to ask your friends and family what they like the most about you what would they say?

What are your hobbies and interests?

Tell me about a typical day in your life

\section{Advice:}

How do you wish able bodied people would treat you?

What do you think the etiquette is for interacting with people who have CP?

Do you want them to acknowledge your disability? To what extent?

\section{Closing:}

What haven't I asked that you thought I would ask?

What else do you have to say, that hasn't been said? OR

What else do you want me to know about your experience with CP? 
APPENDIX 2

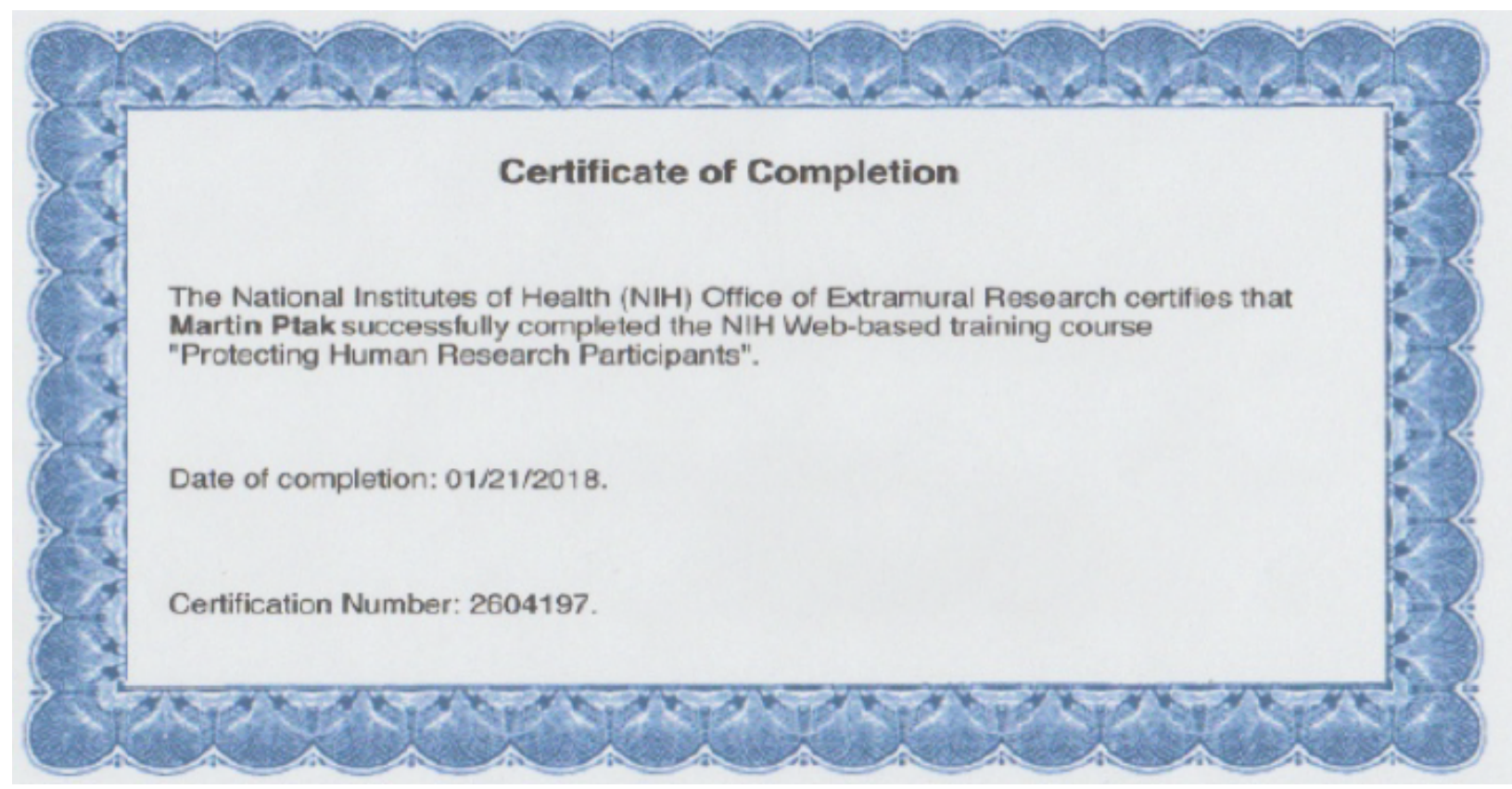




\section{APPENDIX 3}

TCU INSTITUTIONAL REVIEW BOARD

Approval Form

Institutional Review Board (IRB) approval refers to research involving human subjects whether on or off Campus.

Significant changes in design, participants, or measures must be approved by the IRB. Multiyear projects must be submitted annually for approval. Any unexpected adverse effects on human subjects due to procedure should be reported immediately.

Date: February 6, 2018

Principal Investigator: Nina Martin

Project Title: Dancing States as Therapeutic Practice for Performers/Participants with Cerebral Palsy

Multi-year Project: $\quad$ Yes $\quad$ X No

Proposed Participants:

$X$ TCU students, faculty, or staff

$X$ Non-TCU Participants

Special Populations (e.g. children)—specify:

Approval Period: 2/6/18-2/5/19

Board Comments: Amendment (i.e., one year continuing review request; addition of research personnel with NIH training certificates provided) was reviewed by Cathy R. Cox, IRB Chair. This project is minimal risk and approved as expedited in accordance with 45 CFR 46.110 (6)(7).

Approval Number: 1702-021-1802AM

Board Decisions:

Approved, Minimal Risk

$X$ Approved, Expedited

Approved, Exempt Status

Conditional Approval, with following stipulations:

Not Approved for these reasons:

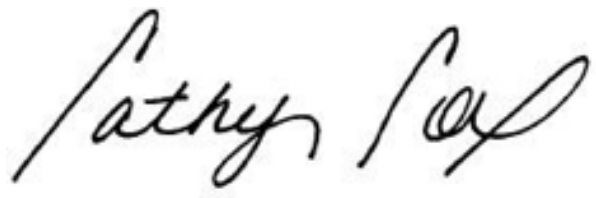

IRB Chair 